University of Pennsylvania Carey Law School

Penn Law: Legal Scholarship Repository

Faculty Scholarship at Penn Law

$1-5-2021$

\title{
Internet Connectivity Among Indigenous and Tribal Communities in North America - A Focus on Social and Educational Outcomes
}

Christopher S. Yoo

University of Pennsylvania Law School

Leon Gwaka

University of Pennsylvania

Muge Haseki

University of Pennsylvania

Follow this and additional works at: https://scholarship.law.upenn.edu/faculty_scholarship

Part of the Communication Technology and New Media Commons, Development Studies Commons, Educational Sociology Commons, Educational Technology Commons, Education Policy Commons, Gender, Race, Sexuality, and Ethnicity in Communication Commons, Indigenous Education Commons, Indigenous Studies Commons, Law Commons, Political Economy Commons, Public Economics Commons, Public Policy Commons, Race and Ethnicity Commons, Rural Sociology Commons, Science and Technology Policy Commons, and the Social and Cultural Anthropology Commons

\section{Repository Citation}

Yoo, Christopher S.; Gwaka, Leon; and Haseki, Muge, "Internet Connectivity Among Indigenous and Tribal Communities in North America - A Focus on Social and Educational Outcomes" (2021). Faculty Scholarship at Penn Law. 2265.

https://scholarship.law.upenn.edu/faculty_scholarship/2265

This Article is brought to you for free and open access by Penn Law: Legal Scholarship Repository. It has been accepted for inclusion in Faculty Scholarship at Penn Law by an authorized administrator of Penn Law: Legal Scholarship Repository. For more information, please contact PennlawIR@law.upenn.edu. 


\title{
Internet Connectivity among Indigenous and Tribal Communities in North America- a focus on Social and Educational Outcomes
}

Christopher Yoo, University of Pennsylvania; Muge Haseki, University of Pennsylvania and Leon Gwaka, University of Pennsylvania

\begin{abstract}
Broadband access is an important part of enhancing rural community development, improving the general quality of life. Recent telecommunications stimulus projects in the U.S. and Canada were intended to increase availability of broadband through funding infrastructure investments, largely in rural and remote regions. However, there are various small, remote, and rural communities, who remain unconnected. Connectivity is especially important for indigenous and tribal communities to access opportunities for various public services as they are generally located in remote areas. In 2016, the FCC reported that $41 \%$ of U.S. citizens living on tribal lands, and $68 \%$ of those in the rural areas of tribal lands, lacked access to broadband, and that the lack of service hindered tribal nations from building their internal structures for self-governance, economic opportunity, education, public safety, and cultural preservation. It is significant to study the indigenous and tribal communities to bridge the digital divide and bring transformative change to these communities. Our findings show some geographic, economic, technological, and generational challenges for connectivity, some unique uses of connectivity for cultural practices, and its impact on educational outcomes in these communities. Since the inception of Southern California Tribal Network, high school graduation has become radically more attainable in these communities due to additional resources made available via the Internet. Connected North promoted interactive Internet learning for students in remote regions to combat endemic local social issues such as a low graduation rate of approximately $20 \%$, and the highest global per capita suicide rate among youth. The contribution of this study is three-fold. First, we identify the geographic, economic, technological, and political challenges for reliable connectivity among indigenous and tribal communities. Second, we show how some tribes and advocacy groups are handling the connectivity themselves, such as operating community networks and remote educational services. Third, we provide an overview of potential impacts of broadband, primarily at the social and educational levels among indigenous and tribal communities. This study suggests approaches for more sustainable connectivity facilities to remote Indigenous communities and reinforces the need for community-driven initiatives to ensure connectivity is conducted in a culturally and technically appropriate manner.
\end{abstract}

Keywords: broadband, deployment, tribal communities, rural, California; Keywords: telepresence technology, education, digital literacy, indigenous, rural, Canada 


\section{Introduction}

This study focuses on the social and educational outcomes linked to connectivity initiatives in tribal communities. The study discusses two connectivity initiatives in tribal communities, Connected North in Canada, and the Southern California Tribal Digital Village (SCTDV) in the United States of America, and their contributions to social and education outcomes in the communities. The study's focus on connectivity initiatives in tribal communities is prompted by two main trends: firstly, the persistent connectivity gaps among resource-constrained communities including tribal communities (Maitland, 2018) and secondly, the urgent need to provide universal affordable connectivity in resource-constrained communities (ISOC, 2020) as well as growing commitment among governments and non-government entities towards addressing these connectivity gaps. These include the US government's stimuli/relief bill setting aside funds for tribal broadband development and activities like ISOC's annual indigenous connectivity summit.

Across the globe, tribal communities have a long history, and in most countries are formally recognised based on different legislation. However, in most instances tribal communities are governed by tribal governments. Lucero, Emerson, Beurle, and Roubideaux (2020) indicate that "sovereign nations determine their own governance structures, laws, and collaborations, including research and research priorities" In the U.S. there are 567 federally recognized Indian Nations that are ethnically, culturally, and linguistically diverse. About 229 of these nations are in Alaska, while others are in 35 other states. Existing studies show that most tribal communities are situated in geographic places with limited resources, far from critical services and face many challenges (Jain, 2018). Further, over the past years, development efforts have been limited resulting in tribal communities lagging in infrastructure including Internet /connectivity infrastructure. In support of this, the Federal Communications Commission (FCC) reports that $27.7 \%$ of Americans in tribal lands lack coverage from fixed terrestrial $25 / 3$ Mbps broadband, as compared to only $1.5 \%$ of Americans in urban areas (FCC, 2020:18). Many factors contribute to the current state of Internet access among tribal communities. Among these, location, population density, and poverty remain the main barriers for Native Americans to be connected (Andrew \& Petkov, 2003).

However, governments and other actors have realised the urgent need to address the connectivity gaps in tribal communities and are pulling together as well as committing substantial resources (O'Leary, Huber, Moran, \& Pesicka, 2019). In the United States, addressing connectivity in tribal communities is a top priority and from 2010 to 2014, the federal government provided more than US\$ 33 billion to promote greater access and adoption of high-speed Internet in underserved areas, including tribal lands, through the FCC's Universal Service Fund and the U.S. Department of Agriculture's Rural Utilities Service. In addition to this, additional funds are being availed in the relief bill while the federal government is redressing spectrum licencing to improve service in the underserved communities. However, government efforts to address connectivity in tribal communities, or any challenge, often take long due to bureaucracy (Gwaka, May \& Tucker, 2018). In many instances, the private sector has contributed to address many challenges but private organisations attempting to address challenges in tribal communities face 
several complexities mainly navigating sovereign governments and securing permission to develop digital infrastructure (setting towers) on tribal/sacred lands.

The prevalence of community-based networks means that many tribes and advocacy groups are opting to handle the matter moving towards handling connectivity matters themselves (Rey-Moreno et al., 2015). Thus, tribal communities, in partnership with different stakeholders, are setting up and operating connectivity initiatives to offer broadband service, building infrastructure, and collecting data for tribal broadband penetration and use. They are taking advantage of advances in technologies and a growing movement towards community-based connectivity initiatives (Plagemann, Canonico, Domingo-Pascual, Guerrero, \& Mauthe, 2008). Initiatives like the Southern California, Connected North and Zenzeleni (in South Africa) are examples of underresourced but innovative connectivity options to provide Internet access in tribal communities. This study only focuses on SCTDV and Connected North as Zenzeleni has received decent attention and is in a different region. The presence of connectivity initiative suggests that even though there are many challenges, it is possible to provide access to tribal communities. However, this study's main thrust is that beyond connectivity, it is important to establish the impact which these connectivity initiatives have on development dimensions (health, education, social) in the tribal communities. available studies indicate that little is known about the impacts of connectivity initiatives such as SCTDV and Connected North on different development dimensions (Howarth et al., 2018).

Connectivity is especially important for tribal communities to access opportunities for growth, productivity, and innovation, as they are generally located in remote areas. The Federal Communications Commission (FCC) reported that the lack of service hindered tribal nations from building their internal structures for self-governance, economic opportunity, education, public safety, and cultural preservation (FCC, 2012). The COVID19 pandemic put to the fore the importance of connectivity as learning and other work-related tasks (and social activities) moved online. Jain (2018:381) indicates that there are "critical gaps between tribals and others in terms of health, education, income and employment opportunities, livelihood and infrastructure provisions" Since education and social dimensions were among the most impacted dimensions, this study focuses on connectivity and the social and education dimensions in tribal communities. Our selection of the development dimensions i.e., social and education dimensions is intentional in that these dimensions are intertwined in many ways. Through connectivity, education practices (and education spaces) are transformed which in turn can influence the social outcomes of children going through the education system. In the wake of COVID19, the health dimension is important and timely to explore but this study is limited to pre-COVID19 evidence.

In this study, we attempt to address the following questions: 1) How are the indigenous and tribal communities addressing their connectivity challenges, 2) What are the informational practices and values among indigenous and tribal communities, and how do those practices and values shape their perceptions towards and use of connectivity? and 3) What impact do the connectivity initiative have on the social and economic dimensions of the tribal communities? Our study follows a case-study approach drawing 
on two in-depth case studies on tribal connectivity: Connected North (Canada) and Southern California Tribal Digital Village (USA). Connected North initiative aims to augment educational and healthcare services to youth in remote indigenous communities in Northern Canada while Southern California Tribal Digital Village initiative is a tribally operated network serving up to 19 federally recognized Southern Californian Indian tribes. This study is within the scope of TPRC as it addresses issues important to different stakeholders within tribal communities including tribal government (tribal leaders/council), tribal community, research funding organisations, researchers.

\section{Research methods}

This study is developed from the 1 World Connected project, an initiative focusing on identifying, analysing, and understanding connectivity initiatives across the globe. To date, the project has identified more than 1,100 connectivity initiatives on different development dimensions (e.g., health, education, and financial inclusion) implemented in different communities, rural, urban, and in tribal communities. From these case studies, this study focuses only on tribal communities stemming from

The study adopts a qualitative study approach. The data collection process started with searching for connectivity initiatives in popular ICT4D databases including the IEEE. Following the identification of these case studies, the research team reached out to the project team for profiling using a standard format questionnaire. This profiling was further supported with engagements with the project teams. In addition to this, the project profiles were updated through desk-based research and participation in open events such as webinars featuring the projects.

The research method applied in this study is appropriate for the study as it allows for detailed descriptions of the case studies. In addition, studying tribal communities is challenging (Harding, Harper, Stone, O’Neill, Berger, Harris, \& Donatuto, 2012) and unpacking detailed aspects takes long. However, our approach allows us to provide basic level descriptions in a comprehensive way and examine changes in specific dimensions (education and social outcomes). There are other social outcomes which take long to manifest, and this presents a potential limitation of the study.

\section{Description of case studies}

This section describes the two case studies used in the study. Even though the case studies are implemented in relatively similar contexts in that both are in tribal communities and in the same geographic region, North America, there are several differences at organisational level. To provide a comparable description, we explore the case studies by examining the technology, organisation, and environment elements. Using this approach, we describe the technology used in each case study followed by the organisation level factors and end with the environment. Beyond this study, framing the case studies in this way simplifies attempts to compare with other existing tribal connectivity initiatives. Even though there are many connectivity initiatives in tribal 
communities, limited case studies have been profiled in comparable formats. In part, this can be attributed to complexities of accessing the tribal communities.

\section{Connected north}

Connected North is a connectivity initiative in the Nunavut territory, Canada's northernmost territory. The territory is almost exclusively made up of about 37,000 indigenous people and is a conglomeration of 25 communities stretching across one-fifth of Canada's total landmass. Infrastructure in and between these communities is poor. There are no roads connecting the communities and telecommunication services in the region are nearly non-existent. When available, Internet is costly and slow further reinforcing the area's isolation. Internet service providers (ISPs), advocates, and the territory's government agree that greater investments in the region's infrastructure are required to better link it with the south. Currently, the government provides connectivity to the region as the few private offerings are unaffordable to Nunavat's citizens. Due to the lack of telecommunications infrastructure, digital literacy and adoption are low in Nunavut as well.

The Connected North initiative is an immersive, digital education, and mental health and wellness network that provides customized services and real-time experiences to students in remote Indigenous communities. It deploys Cisco's Telepresence technology (MX 300 G2) to offer real-time, live interactive experiences with experts and students in other classrooms around Canada. Launched as a pilot in a single school, the project has grown with current implementation in 65 schools with plans to deploy telepresence units in more than 100 schools. The project's expansion, while desperately needed, is curtailed by cost and funding concerns.

In smaller schools, telepresence setups are put in common areas so all classes may use them as needed. In larger schools, each intermediate classroom $\left(6^{\text {th }}, 7^{\text {th }}\right.$, and $8^{\text {th }}$ grades $)$ has a unit. Cisco was selling these units for CA $\$ 30,000$ (US\$23,611.82 but they offered a 70 percent discount to Canadian school districts, bringing the cost down to approximately CA $\$ 9,000$ (US\$ 7,200). Due to the program's success, Cisco spun-off Connected North to be managed and led by a charity partner, TakingITGlobal. Together with Cisco, the team built an ecosystem of more than 50 funding partners including federal, provincial, and territorial governments, private sector lenders, private foundations, and individual donors.

Connected North's success is indicative of the utility of immersive, interactive Internet learning for students in remote regions to combat endemic local social issues such as a low graduation rate of approximately 20 percent, and the highest global per capita suicide rate among youth. The impetus and continued support for Connected North comes from two tragic statistics of Canadian indigenous youth: the dropout rate is approximately 80 percent, and these communities have the highest rates of youth suicide per capita in the world. Combatting these systemic problems required more than just educational resources. Connected North provides students with access to remote mental health and wellness professionals that are otherwise inaccessible. The continued success and 
expansion of the Connected North project is shows feasibility of technological interventions to ameliorate systemic social issues in remote communities. While it may not be possible to provide experts and healthcare providers to remote populations physically, Cisco's telepresence units help bridge coverage gaps.

There are five overarching content areas that Connected North focuses on: virtual fieldtrips, mentoring, experts on demand in a vast array of subject areas, classroom cultural exchanges (between indigenous and non-indigenous student populations and among indigenous classrooms), and programming called "Future Pathways," which aims to help students attend post-secondary institutions as well as make them aware of career paths that are available to them. Furthermore, 90 percent of the content is indigenous themed. Connected North staff work together with teachers for content offerings, and work to reinforce indigenous cultural educational styles, such as the emphasis on collaborative learning in Inuit schools.

\section{Southern California Tribal Digital Village}

The Southern California Tribal Digital Village (SCTDV) is a connectivity initiative administered by the Southern California Tribal Chairmen's Association (SCTCA), a nonprofit serving 19 federally recognized Southern Californian Indian tribes since 1972. The Tribal Digital Village, and early community network, was established in 2001 with the goal of serving the needs of its tribal members and descendants in the San Diego County urban areas by bringing Internet access to Native American communities. In terms of partnerships, the SCTDV is supported by the private sector and academic institutions including HP, Microsoft, University of California, Cal State Libraries. The initiative offers training on awareness generation about the benefits of broadband.

The Southern California Tribal Digital Village was developed using a mix of technologies which include broadband wireless, TV white space, and fiber. In terms of power options to power the technologies, the initiative relies on alternate energy sources, thus, it is solar powered. The network operates as a high-speed wireless microwave network, with some experimental forays in TV white space (TVWS) and has an eye towards incorporating fiber optics where possible. The operations team aims to keep up with evolutions in technology and network capability.

The network serves a collection of tribal communities and is focused on social welfare and educational outcomes. Since the network's inception in 2001, high school graduation has become radically more attainable in these communities due to additional resources made available via the Internet. By providing free Internet access to libraries, schools, head start programs, and health and community centers, SCDTV prioritizes the support and empowerment of its users. However, the SCDTV provides a paid service to private homes on a sliding scale between US\$20, US\$ 35 , and US\$ 64,95 . This assures both that the initiative is sustainable profitability and encourages the integration of all kinds of digital tools and cultures into tribal communities.

In terms of the project's way forward, the project began somewhat short-sightedly, operating on a grant-to-grant basis before transitioning to a sustainability-based 
business plan. The SCTDV team notes that immediate emphasis on financial planning and long-term maintenance would have better served their aims. In addition to this, the project team is targeting to implement a network-by-network planning. Like many other successful network builders, SCTDV highlights the importance of considering the unique needs and goals of each individual network. It is key to begin with a clear knowledge of each network's specific end goals and outcomes, as well as throughout knowledge of both terrain as well as the requirements and priorities of the community the network is meant to serve.

\section{Challenges for reliable connectivity among indigenous and tribal communities}

In the setup and operation of the two case studies described, there were many challenges faced. Some of the challenges faced are like common challenges experienced in other connectivity initiatives not in tribal communities. This section expands on the geographic, economic, technological, and political challenges. However, we attempt to highlight challenges that are unique to tribal communities. Were possible, the section also explains the mechanisms which different actors have developed to overcome the challenges.

Tribal communities are characterised by vast lands with sparse population and households with low income. Much of the tribal land in this area is limited to mountainous areas. Additionally, the borders of tribal lands are discontinuous, often interrupted by county lands. Focusing on the SCTDV, the geography of canyon requires unique technologies that do not rely on the line of sight and such technologies are often costly. The unique geographic and socioeconomic characteristics make it difficult to develop appropriate connectivity models. Conventional connectivity models are not ideal when addressing connectivity needs of such communities. To address this. The SCTDV combines different technologies but this increases the capital outlay and the operating costs.

In additional to these technical aspects, there are also sociocultural factors which may impede connectivity initiatives. firstly, there are cultural rules which must be observed when setting up infrastructure (towers) on tribal lands. Tribal governments must authorise (or be consulted) when installing towers. This often complicates initiatives by governments or the private sector. Secondly, there is a growing scholarship and debates on spectrum allocation. Spectrum is recognised as a resource and rights of tribes to spectrum over tribal communities must be observed. However, even when tribes are allocated spectrum, most lack adequate financial resources to utilise the spectrum often pushing governments to engage establish telecommunication companies.

The third sociocultural aspect relates to the generation gap. Tribal elders can prove resistant to the introduction of technology, fearing it will threaten the preservation of their communities, history, and culture. Such resistance is more pronounced when the project implementing team consists only of outsiders. To address this, the Connected North initiative pays attention to indigenous cultural specificities by ensuring that the telepresence unit function is congruent with Inuit pedagogies about collaborative 
learning and mixed-media lessons. In the project's present incarnation, local curricula guide the type of content that Connected North makes available.

In addition to the foregoing challenges, limited financial resources are also a major challenge when connecting tribal communities. The development and operation of connectivity interventions requires capital and operating expenses. capital expenses for the Connected North initiative were covered by CISCO through a CSR initiative. CISCO continues to support Connected North with operating expenses (cost to stream sessions). However, such models are often not sustainable. For the Connected North project are easily scalable. Yet, the largest impediment to rolling out telepresence units in more school districts is operating cost. Cisco contributed more than US\$ 5 million, and while donors and governmental entities have been generous, yet funding still falls short of providing units to all the schools who desire them. It is estimated that a 45-minute live session with an expert guest speaker or Indigenous Role model for classrooms in Northern communities' costs $\$ 200$. So far, the private sector has supported the program through donations.

The SCTDV also faces financial challenges, albeit, with a difference. In the United States, the FCC provides funds to support the broadband initiatives in tribal communities. However, In the U.S., federally recognized indigenous tribes have a relationship of sovereign dependence to the government and not states. This status denotes a complex political status; whereas sovereignty signifies self-governance and independence, 'dependence' indicates tribes' subordination to federal regulations and laws. When coupled with chronic governmental inattention to the needs and rights of Native American tribes, this status can prove to be a stumbling block to pursuing grant funding. For example, when the federal government outsources funding decision to state governments, tribal eligibility is threatened because "dependence" delimits tribes' political relationships narrowly to the federal government. Certain instances of legislative oversight and targeted exclusion have been successfully sidestepped by helpful partnerships with private companies and public universities.

The lack of connectivity in tribal communities persist due to geographic, economic, technological, and political challenges. However, there is progress through different initiatives. In this section, we explored two initiatives implemented in North America, CN and SCTDV. While there are some similar challenges between the initiatives, there are also unique ones. There are mechanisms in place to address these challenges for instance, some tribes and advocacy groups are handling the connectivity themselves, such as operating community networks and remote educational services. in the next section, the study turns to explore the outcomes of the initiatives in the tribal communities.

\section{Social and educational outcomes}

So far, we have described the two case studies focusing on the technology, organisation, and environment aspects. We highlighted some of the challenges being faced. In this section, building on the realisation that, within the ICT4D discipline, there is a growing need to understand/demonstrate the benefits associated with the interventions to the 
communities in which they are implemented (Mthoko \& Khene, 2015), we focus on the outcomes linked to the initiatives. With government and non-government parties contributing towards the development of infrastructure in tribal communities, it is important to establish the contribution of these investments towards the needs of the communities. Exercises to assess the outcomes linked to interventions are common for instance, the National Commission on Libraries and Information Science 1997 undertook an exercise to understand how connected libraries could meet the needs of the communities. In the context of this study, even though prior studies suggest that connectivity can play many roles in tribal communities, producing such evidence. Kaufman et al. (2016) suggest that technology, including connectivity, might be helpful in adolescent pregnancy prevention programs. In this section, we explore the social and educational outcomes of the two initiatives.

\section{Educational outcomes}

Education in tribal/indigenous communities, like other development dimensions (health), has been poor. Governments have recognised this and embarked on series of actions to address this. In the United States, an executive order was issued aiming to improve the educational opportunities provided to tribal communities. The Tribal Educational Framework attempts to develop a concrete pathway creating a Cradle-toCareer Pipeline: this framework focused on the idea - path early education in preparation for - college - career. In Canada, Ottman (2017) identifies education gap between Aboriginal and non-Aboriginal and argues that education among the Aboriginal students needs a revamp to make way for inclusive, innovative, caring, and supportive spaces in education.

The SCTDV and Connected North initiatives allow education to occur in the tribal communities. The Connected North initiative provides students with an immersive learning experience which allows students to experience other environments without leaving their community. Existing studies demonstrate the importance of balancing education and maintaining cultural and historical connections. tribal communities have an opportunity to learn their Native languages and histories in addition to receiving a complete and competitive education. In addition to students within the communities, connectivity initiatives also allow college students to come back during breaks and continue learning activities online. The importance of this possibility was evident in the context of Covid. Even though there are some similarities in benefits obtained by the tribal communities from the connectivity initiatives, some are community specific. We explore how each intervention impact education and social outcomes in the communities in which they are implemented.

\section{Connected North and education}


The Connected North project began in 2013 with a single pilot school. As of 2020, there are currently 65 schools, providing almost 3,000 sessions reaching more than 15,000 students. Other locations where telepresence units are in use include the Northwest and Yukon Territories, and the province of Ontario. The project is targeting to connect at least 100 schools in the next five years. The Connected North initiative partners only with those communities who request the service, and many school districts have indicated interest and are currently on a waiting list. In Nunavut, the territory where the pilot school was located, the government asked Connected North to rollout the service in all its 43 schools, which they will subsidize as a budget line item.

Despite experiencing challenges at the start, schools averaged three to four sessions a week by 2017. During the interactive sessions, students engage with external role models and inspirational individuals. As such, the learning activities extend beyond basic education to include activities about other cultures and social practices outside of their own communities. In addition, there are sessions dedicated to counselling to address mental health related challenges within the communities.

Connected North has continually relied on feedback from students and teachers in schools with the telepresence units, and the response has been uniformly positive. reflecting on the impact of Connected North initiative on learning, teachers reported net positive gain on student engagement and success. In the pilot deployment, 81 percent of students reported that they learned more in a telepresence session than in their traditional classroom coursework. In addition, students found telepresence sessions made science more interesting (83 percent) and more enjoyable (89 percent). Overall, assessments echoed positive impact of the connectivity initiative on student interest level.

In addition, evaluations of the 2015-2016 academic year, for instance, found that 60 percent of the students found the immersive sessions interesting and wanted to learn more. Connected North's intervention has also positively impacted individual students' views on their future. The study found that sixty-four percent of the students believe they can be successful in the future, and 62 percent are excited about their futures. The telepresence units also empower teachers, along with the students. Connected North works in synchronization with teachers to provide the materials that are most relevant to the educators' lesson-plans. Also, the organization offers a considerable amount of professional development opportunity for faculty.

\section{Southern California Tribal Digital Village and education}

In the 19 communities connected, upwards of 500 homes are currently connected. The network also incorporates libraries, schools, head start programs, and municipal centers. The program is actively growing; with a total of roughly 10,000 residents in the areas they serve, they currently estimate their reach is between 1,500 and 2,000 users and aim to expand access to an additional 1,000 people in the near future.

In terms of education, access to the Internet has contributed towards lowering high school attrition rates. Since 2001, high school graduation has become radically more 
attainable in these communities due to additional resources made available via the Internet. In communities that often require consecutive missed school days for students to tend to cultural and family responsibilities, after-school programs and learning software such as Cyber High and PLATO have allowed students unprecedented opportunities to stay abreast of their curricula. With less students falling behind, more remain flexibly engaged with their studies and graduate, empowering young people as well as strengthening communities.

In most tribal communities, tribal colleges, and universities. However, other opt to access different type of education e.g., learning about new cultures and the outside world. Prior to the setup of the connectivity initiatives, individuals would have to leave their communities to experience this elsewhere.

\section{Social outcomes}

In addition to educational outcomes, this study explores the social outcomes related to the two connectivity initiatives in the tribal communities they are implemented. Even though there is a direct link between the connectivity initiatives and social outcomes, there are also indirect impacts. For instance, changes in education (which are made possible through the connectivity initiatives) can have social outcomes (Appiah \& McMahon, 2002).

In both communities, students were found to be particularly interested in sharing their own culture and learning about geographically diverse peers' cultures, and telepresence units are particularly suited to facilitate this. In the Connected North context, the immersive experience is designed to promote virtual interaction and engagements between the students and outside experts. In this way the telepresence provides and opportunity for students to demonstrate their everyday practices (including cultural) as well as experience a new culture, albeit virtually. Closely related to this, the telepresence teaches young people life skills which enable them to progress in life and provide for themselves.

Even though the virtual experiences are allowing students to share their culture and learn about different culture, there are concerns especially among the elderly that young people are being exposed to "foreign cultures" which may also mean exposure to inappropriate images. Digital technologies associated with connectivity raise concerns among the cultural leaders including that external people want to gain access to the "hidden dynamics" of the tribes. However, in this study we found that the Connected North initiative pays a lot of attention to indigenous cultural specificities. The way in which telepresence units function is congruent with Inuit pedagogies about collaborative learning and mixed-media lessons. In the project's present incarnation, local curricula guide the type of content that Connected North makes available. 
Connectivity also plays an important role in the health dimension. Many studies on health and technology focus on application of tech (mhealth). In this study, we found that immersive technology played a key role towards addressing mental health problems. In Canada, suicide rates among the indigenous groups are estimated to be three times higher than that among non-indigenous people. CISCO highlighted that the Connected North project was developed in part to address the suicide challenge among the indigenous groups. The Connected North initiative has enabled young people to have access to counselling services. In addition, the initiative allows individuals to acquire knowledge (learn) about new cultures/general education without "removing themselves" from their communities that reduces stress. Apart from mental health issues, the connectivity initiatives provide students opportunities to learn about the global health issues when they engage health experts. During

\section{Discussion}

This study aimed to discuss the impacts of connectivity initiatives on educational and social outcomes in two tribal communities. The study started by describing two initiatives connectivity initiatives, Connected North and SCTDV implemented in tribal communities. This demonstrates the current efforts to connect tribal communities even though there are many other tribal communities which remain unconnected. In describing the two case studies, the study demonstrates that efforts to connect the unconnected tribal communities are encounter additional unique challenges in addition to the common challenges faced when connecting other resource-constrained communities. For instance, in tribal communities, sovereignty and cultural lore may have implications on issues such as spectrum allocation and setup of connectivity boosters on tribal land.

This consideration is relevant and timely in the context of the United States were FCC is inviting competitive bids for spectrum allocation in tribal communities. Lesson from the study suggest that it is important to ensure that communities are included and approve of the efforts by external players to develop connectivity infrastructure. Tribal communities must be included as owners through co-sharing agreements. Lessons from the community network deployments over the past years suggest that connectivity initiatives model promoting community participation achieve considerable sustainability and success.

Further, the study findings demonstrate the importance of economic sustainability planning. In the Connected North project, live sessions continue to be sponsored through donations e.g., CISCO conducts fundraising to sustain the initiative. Findings from the two initiatives suggest that local communities are provided access to connectivity at low and/or no cost. This means the initiatives must secure funds from elsewhere to continue operating. However, this was noted as a major setback in terms of the expansion and operations of the initiatives. studies on connectivity initiatives indicate that in most resource-constrained communities, Internet access is provided for no charge, but this is not a sustainable practice. Kuriyan et al. recommend that even when providing Internet access to the least privileged communities, it is critical to build in economic sustainability 
practices. In the study, we found that the initiatives can continue the operations through support from multiple stakeholders and to an extent, limited revenue generation options. At the same time, the study has highlighted the plight of the households in the tribal communities suggesting that the households have low income making it difficult to charge them for connectivity. Developing a balance on affordable access becomes a critical. In this regard, the study highlights that conducting extensive studies of different connectivity initiatives in different tribal communities might be critical towards the efforts to identify an optimal model.

In terms of the education outcomes, Chigona (2017) suggests that initiatives must consider the learner's background as students from different communities (in this instance, tribal communities), have different needs. In the same context, when assessing the education outcomes, it might be important to frame these based on local context. In this study, Connected North immersive considered the circumstances of the students. MacKenzie (2009:371) indicates that "An education system which does not take into account local culture and language is characterised by low intake, high repetition and high dropout rates". In this regard, the immersive technologies used in the Connected North case were aligned to the culture and local context.

From our study, there are several avenues for further work. First, additional work can be done to understand the impacts of the connectivity initiatives on other wellbeing dimensions to augment our findings on the educational and social outcomes focused on in this study. We briefly discussed the impacts of the connectivity initiatives on health relating to the Connected North initiative, but additional work might shed more light on the usefulness of the connectivity initiatives in the context of Covid19. In addition, comparing only two case studies might produce limited insights. There are efforts to increase connectivity initiatives in tribal communities and increasing the number of case studies compared might produce new useful insights.

In addition, there are also other critical issues to be addressed among tribal communities. These include growing concerns such as tribal communities could be among the "news deserts" with local activities not being reported in the mainstream media (Thorson et al., 2020). In this regard, providing connectivity might serve as an opportunity to bring to the fore activities within the tribal communities.

\section{References}

Adler, M. A., \& Wilshusen, R. H. (1990). Large-scale integrative facilities in tribal societies: Cross-cultural and southwestern US examples. World Archaeology, 22(2), 133-146. 
Andrew, T. N., \& Petkov, D. (2003). The need for a systems thinking approach to the planning of rural telecommunications infrastructure. Telecommunications Policy, 27(12), 75-93.

Appiah, E. N., \& McMahon, W. W. (2002). The social outcomes of education and feedbacks on growth in Africa. Journal of Development Studies, 38(4), 27-68.

Burman, B. R. (2003). Indigenous and tribal peoples in world system perspective. Studies of Tribes and Tribals, 1(1), 7-27.

Chigona, A. (2017). Western Cape Subject Advisors' Perception of Their Preparedness for Connected Classrooms. Electronic Journal of e-Learning, 15(5), 444-454.

Gwaka, L. T., May, J., \& Tucker, W. (2018). Towards low-cost community networks in rural communities: The impact of context using the case study of Beitbridge, Zimbabwe. The Electronic Journal of Information Systems in Developing Countries, 84(3), e12029.

Harding, A., Harper, B., Stone, D., O’Neill, C., Berger, P., Harris, S., \& Donatuto, J. (2012). Conducting research with tribal communities: Sovereignty, ethics, and data-sharing issues. Environmental health perspectives, 120(1), 6-10.

Howarth, A., Quesada, J., Silva, J., Judycki, S., \& Mills, P. R. (2018). The impact of digital health interventions on health-related outcomes in the workplace: a systematic review. Digital health, 4, 2055207618770861.

ISOC. (2020). Indigenous Connectivity Summit 2020. Accessed December 24, 2020 from https://www.internetsociety.org/events/indigenous-connectivity-summit/2020/

Jain, S. (2018). Promoting tribal equity through public expenditure in low income states. UPUEA Economic Journal, 14(1), 381-384.

Kaufman, C. E., Schwinn, T. M., Black, K., Keane, E. M., \& Big Crow, C. K. (2016). The promise of technology to advance rigorous evaluation of adolescent pregnancy prevention programs in American Indian and Alaska Native tribal communities.

Lucero, J. E., Emerson, A. D., Beurle, D., \& Roubideaux, Y. (2020). The holding space: A guide for partners in tribal research. Progress in Community Health Partnerships: Research, Education, and Action, 14(1), 101-107.

MacKenzie, P. J. (2009). Mother tongue first multilingual education among the tribal communities in India.International Journal of Bilingual Education and Bilingualism, 12(4), 369-385.

Maitland, C. F. (2018). Now you see it, now you don't: Digital connectivity in marginalized communities. Computer, 51(6), 62-71.

Maldonado, J. K., Shearer, C., Bronen, R., Peterson, K., \& Lazrus, H. (2013). The impact of climate change on tribal communities in the US: displacement, relocation, and human rights. In Climate change and indigenous peoples in the United States (pp. 93-106). Springer, Cham. 
Mthoko, H., \& Khene, C. (2015, May). Assessing outcome and impact: towards a comprehensive evaluation approach in ICT4D. In Proceedings of the Seventh International Conference on Information and Communication Technologies and Development (pp. 1-4).

O'Leary, R., Huber, C., Moran, A., \& Pesicka, J. (2019). Developing the Tribal Resource Guide and the Poverty and Culture Training: The We RISE (Raising Income, Supporting Education) Study. American Indian and Alaska native mental health research (Online), 26(2), 134-150.

Ottmann, J. (2017). Canada's Indigenous peoples' access to post-secondary education: The spirit of the 'New Buffalo'. In Indigenous pathways, transitions and participation in higher education (pp. 95-117). Springer, Singapore.

Plagemann, T., Canonico, R., Domingo-Pascual, J., Guerrero, C., \& Mauthe, A. (2008). Infrastructures for community networks. In Content Delivery Networks (pp. 367-388). Springer, Berlin, Heidelberg.

Rey-Moreno, C., Sabiescu, A., Siya, M. J., \& Tucker, W. D. (2015). Local Ownership, Exercise of Ownership and Moving from Passive to Active Entitlement: A practice-led inquiry on a rural community network.

Thorson, K., Medeiros, M., Cotter, K., Chen, Y., Rodgers, K., Bae, A., \& Baykaldi, S. (2020). Platform Civics: Facebook in the Local Information Infrastructure. Digital Journalism, 8(10), 1231-1257. 